\title{
A spectacle-mounted liquid-crystal tachistoscope
}

\author{
P. MILGRAM \\ University of Toronto, Toronto, Canada
}

\begin{abstract}
A spectacle-mounted shuttering device, the Portable Liquid-crystal Apparatus for Tachistoscopy via visual Occlusion, based on light-scattering cholesteric liquid crystals, is introduced. Operating characteristics of the device are discussed with respect to the design objectives of comfort, switching speed, light transmission, light extinction, safety, and reliability. A number of practical research, clinical, and industrial applications of the spectacles as tachistoscope, stroboscope, and stereoscopic viewing apparatus are summarized.
\end{abstract}

In its conventional form, a tachistoscope is basically an enclosed container, made of some lightproof material, into which an observer looks and in which, by means of special lamps, it is possible to illuminate specific objects within the container. By using a suitable optical system, one can present visual information in a precisely controlled manner to one or both eyes of an observer. To qualify as a tachistoscope in the literal sense, such a viewing apparatus must be capable of allowing very rapid presentation of visual information. (The word tachistoscope derives from a combination of the Greek words takhistos ["swiftest'] and skopeo ["look at"].) Often, instead of enclosed containers and special lamps, computer display screens or (computer-controlled) projection devices are used for tachistoscopic experiments (Green, 1978). In this paper, the design objectives, implementation, and applications of another type of tachistoscope, a spectaclemounted liquid-crystal shuttering device, are described.

A photograph of a prototype of the electrooptic shuttering device, illustrating both its "open" and "closed" states, is given in Figure 1. The acronym given to the device is PLATO, for Portable Liquid-crystal Apparatus for Tachistoscopy via visual Occlusion. The emphasis on portability stems from the fact that, by wearing such a spectacle-mounted apparatus, the subject in an experiment is not restricted to remaining in front of an enclosed viewing container, or a fixed display device, or a projection screen; thus, a large range of experiments involving ambulatory subjects can be carried out. The reference to "visual occlusion" in the acronym as the means of implementing this tachistoscope derives from the fact that, rather than illuminating or projecting specific target images, as do other tachistoscopes, the PLATO device regu-

The device described here was developed at the TNO Institute for Perception in Soesterberg, The Netherlands. The author gratefully acknowledges the coliaborative efforts of S. Burrij, Y. Hartmann, R. Ohmann, R. van der Horst, and P. Bakker of TNO; the suggestions of D. Reits of the University of Amsterdam Institute of Ophthalmology; and the very valuable information supplied by F. C. Saunders of the UK Royal Signals and Radar Establishment. Address correspondence to P. Milgram, Department of Industrial Engineering, University of Toronto, Toronto, ON M5S 1A4, Canlada. lates image presentation instead by selectively blocking out the subject's view of the target, the illuminance of which remains independent of the state of the shutters.

In the following sections, the background and the design objectives underlying the development of the PLATO device are presented, followed by a detailed description of its properties and, finally, a short discussion of some already demonstrated and further foreseen applications.

\section{BACKGROUND AND DESIGN OBJECTIVES}

The development of the device reported here originated from a need for a flexible instrument that would be capable of occluding the visual field of an automobile driver during selected intervals of time, for the purpose of investigating various aspects of automobile driving behavior, such as visual information processing in different roadway environments and drivers' decision-making strategies under varied driving conditions. (For specific uses, see Applications.) Since the technical requirements for carrying out actual road tests with an instrumented vehicle are in general rather stringent, it soon became clear that any device meeting the design specifications associated with this application would most likely be able to serve also as a general-purpose tachistoscope/visualocclusion device for a much wider range of psychomotor research applications.

The design objectives are listed below, and the extent to which these have been satisfied are described in the following section: (1) The device had to be comfortable, that is, quiet, easy to put on and wear for fairly long periods of time, not physically restraining, and free of any disturbing mechanical vibrations. (2) Activation of the device had to be accurately controllable and rapidly switchable. (3) In its open state, the device had to affect as little as possible the perceived brightness, sharpness, and spectral composition of objects/images being viewed. (4) In its closed state, the device had to block out all pertinent visual information in the subject's visual field, while interfering as little as possible with the eye's performance during subsequent open-state intervals. (5) The device was required to be safe, reliable, and relatively rugged. 


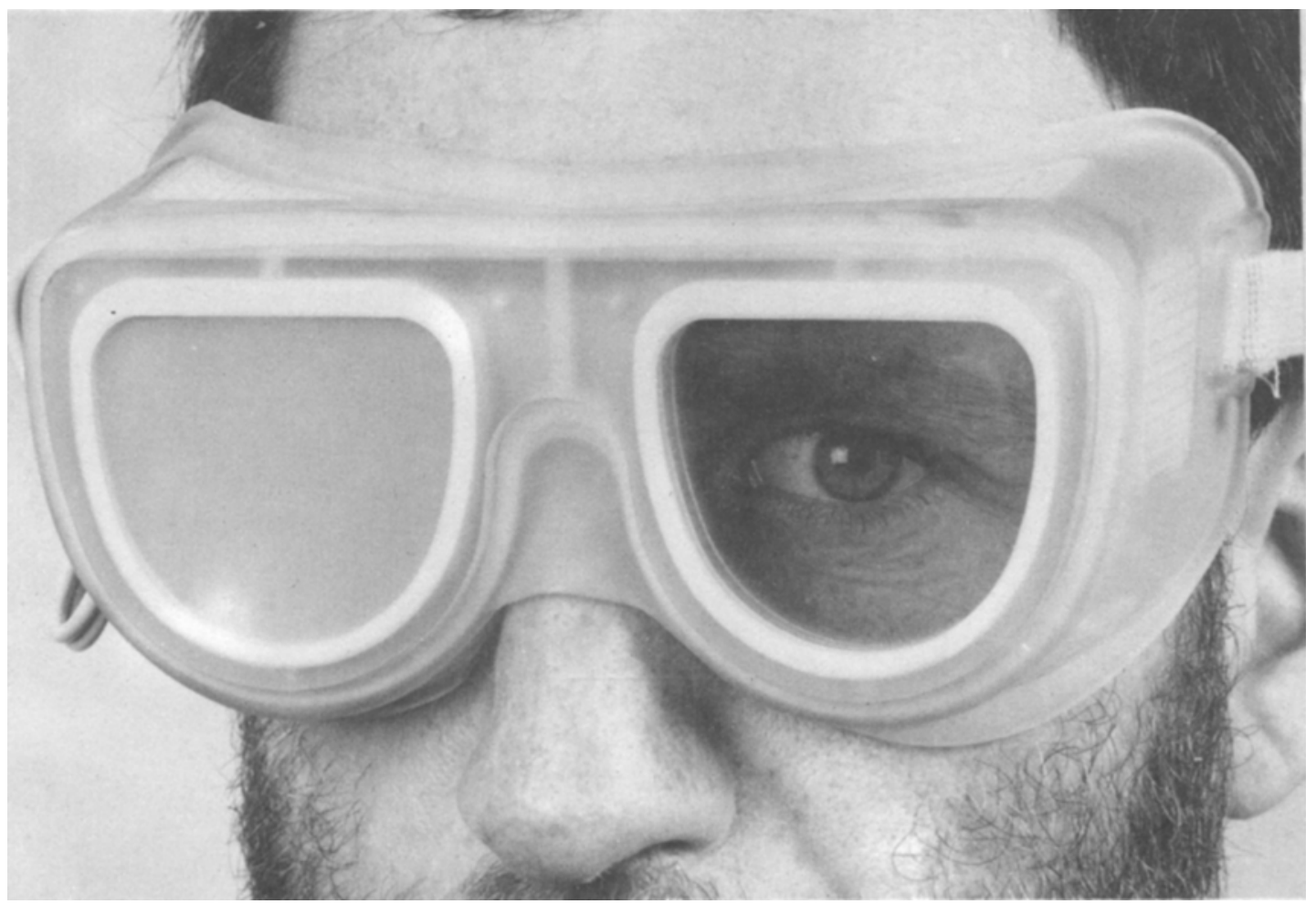

Figure 1. Prototype of PLATO, illustrating both transparent "open" state and scattering "closed" state.

\section{DESCRIPTION AND PROPERTIES}

The solution to the design problem outlined above was to construct a spectacle-mounted liquid-crystal shuttering device, based on the cholesteric-nematic phase change effect (Mollon, Polden, \& Morgan, 1977; Raynes, 1976). Briefly, the device operates as follows: When a sufficiently large electrical field is applied across the electrodes of a liquid-crystal cell comprising a suitable mixture of cholesteric and nematic liquid-crystal materials, the molecules of the mixture align themselves longitudinally in parallel with each other. In this (pseudo-nematic) phase, the cell is transparent and essentially all incident light is transmitted with no perceivable distortion. When the field is removed, or reduced to a subcritical level, the cell quickly relaxes into its cholesteric phase, characterized by a set of molecular helices that are randomly distributed throughout the cell. In this "closed" state, the cell acts to scatter incident light, resulting in a uniformly luminous milky texture. Upon reimposition of the electrical field, the transparent "open" state rapidly reappears. Both of these states are illustrated in Figure 1.1

It is important to point out that the PLATO device operates differently from the majority of other electrooptic shuttering devices, which use crossed polarizers and which are able to vary light transmission continuously between transparent and opaque by rotating the plane of polarization of incident light. Since with the PLATO spectacles incident light is either transmitted or scattered, with essentially no intermediate state, it is more appropriate from the point of view of the observer to consider the mechanism as a "visual information switch" rather than as a light modulator, or "light valve," type of shutter. Further details and operating characteristics of the PLATO spectacles are presented below, with each design objective addressed individually.

\section{Physical Characteristics}

The requirement for the wearer's comfort has been satisfied by the choice of a silent, lightweight electrooptic shuttering device rather than a mechanical shutter. (By comparison, in some earlier visual-occlusion experiments-for example, those of Fraser \& Perry, 1980, and of Senders, Kristofferson, Levison, Dietrich, \& Ward, 1967-pneumatically driven visors mounted on motorcycle helmets were all that was available.) The present PLATO prototypes have been constructed both as ordinary spectacles and as goggles (the latter shown in Figure 1), weighing approximately $50 \mathrm{~g}$ and $200 \mathrm{~g}$, respectively. The principal advantages of the safety goggle model are that the wearer is able to mount these easily over his/her own spectacles and that the wearer's peripheral visual field is also occluded. (If required, a goggle-mounted model with switchable peripheral field lenses is also quite feasible.)

At present, one potential drawback of the device is the necessity of controlling the liquid-crystal cells via a cable leading from a remote power supply. This is clearly 
a constraint on the subject's mobility for some applications, and it also increases the likelihood of tripping over wires, dropping the glasses, and so forth. It is expected, however, that it will eventually be possible to construct a totally battery-powered version, which could then also be remotely controlled, for example by telemetry or infrared light.

\section{Switching Characteristics}

In Figure 2, the transmissive switching properties of a prototype PLATO cell are illustrated. These data (redrawn for convenience from an oscilloscope photograph) were obtained by switching the cell on with a 5msec, $140-\mathrm{V}-\mathrm{rms}, 2-\mathrm{kHz}$ voltage pulse (repeated every $35 \mathrm{msec}$ ). One surface of the cell was illuminated by a collimated beam of light that covered its entire surface area; the luminance output waveform drawn in Figure 2 was obtained with a Pritchard photometer. As shown in the figure, the turn-on time of the cell is approximately $1 \mathrm{msec}$.

It is important to note that the transition from the scattering closed state to the clear open state occurs when a critical level of the electrical field (as opposed to voltage) across the cell is achieved. Since higher threshold fields imply faster cell response times (Raynes, 1976), and since the value of the threshold can be controlled by adjusting the thickness of the liquid-crystal layer and/or the concentration of cholesteric in the liquid-crystal mixture, it is possible to use cell composition and thickness as design parameters to expedite the device's turn-on time.

The exact time taken to return from the open to the closed state is somewhat less straightforward to determine. First of all, note in Figure 2 that there is a time delay, or latency, of approximately $2 \mathrm{msec}$ between cessation of the driving voltage and commencement of relaxation. Although it could be argued that technically this delay time should be considered as part of the turn-off time of the cell, it is clear that for applications involving well-defined open intervals this effect can in practice easily and unequivocally be compensated for simply by designing the switching circuitry to allow the driving voltage to turn off $2 \mathrm{msec}$ prior to the desired end of the open interval. The effective duration of the remaining portion of the turnoff time of the cell is less easy to quantify, however.

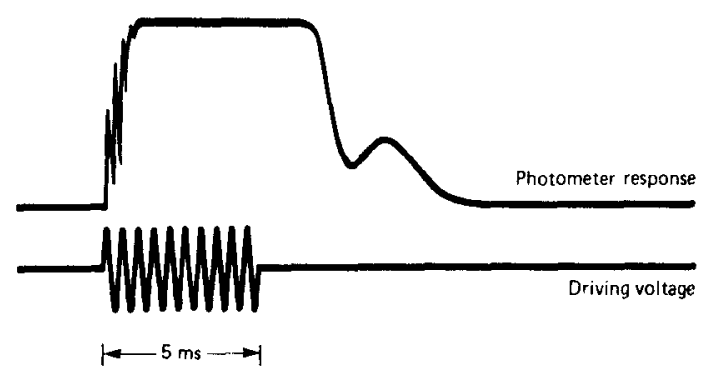

Figure 2. Transmissive switching properties of PLATO cell. Upper trace: Photometer output waveform. Lower trace: Voltage input (140-V-rms, 2-kHz, 5-msec pulse).
Adopting a simple definition of turn-off time, such as the time taken for transmission to fall to $10 \%$ of its maximum level, it would appear from Figure 2 that this time is approximately $4 \mathrm{msec}$. It is important to point out, however, that during the nematic-to-cholesteric phase change-that is, as the liquid crystal cells become progressively foggier, at some point after the start of the relaxation period but before maximum scattering (minimum transmission) is reached-the observer will no longer be able to receive coherent visual information from any images on the other side of the cell. The effective turn-off time of the cell, from the point of view of the human observer, therefore, can be much shorter than the objectively measured relaxation time for pure light transmission. The precise value of this effective turn-off time depends, among other things, on the level of ambient illumination and on the contrast ratio of the visual images being observed, with consequently longer turn-off times for higher contrast images.

\section{Open-State Characteristics}

Looking through the liquid-crystal cells in their clear open state is essentially identical to looking through untinted glass spectacles, as illustrated in Figures 1 and 3. (Note in Figure 1 that, since the walls of the goggles have inhibited external illumination of the eye in the photograph somewhat, the open left eye lens appears here to attenuate light more than is actually the case, as seen comparatively in Figure 3.) For the present cells, each comprising a layer of liquid-crystal material sandwiched between two parallel $1.2-\mathrm{mm}$-thick glass plates coated with a transparent conductive electrode layer of indium tin oxide (resistance $100-300 \Omega$ per square), light transmission is approximately $85 \%$. This high level of light transmission is very important for applications, such as stereoscopic displays described below, for which maximum image brightness is desired.

\section{Closed-State Characteristics}

It is important to emphasize that the light-scattering cholesteric liquid crystals do not become opaque in their closed (off) state, but remain translucent. The subject's eye therefore remains substantially illuminated during the spectacles' closed state and consequently much closer to the level of illuminance present during subsequent open intervals than would be possible with opaque shutters. For applications involving relatively long periods of visual occlusion, this implies that the extent to which the eye must continually adapt to new light levels as the shutters open and close is reduced. For more rapid repetitions (stroboscopy), the issue is less one of repeated adaptation of the eye than of mean level of illuminance, which is clearly higher with light-scattering lenses than with opaque shutters.

The degree of extinction of the shutter in its closed state, in this case extinction of visual information rather than extinction (attenuation) of light, is not simple to measure photometrically. This is because, as mentioned above, the 


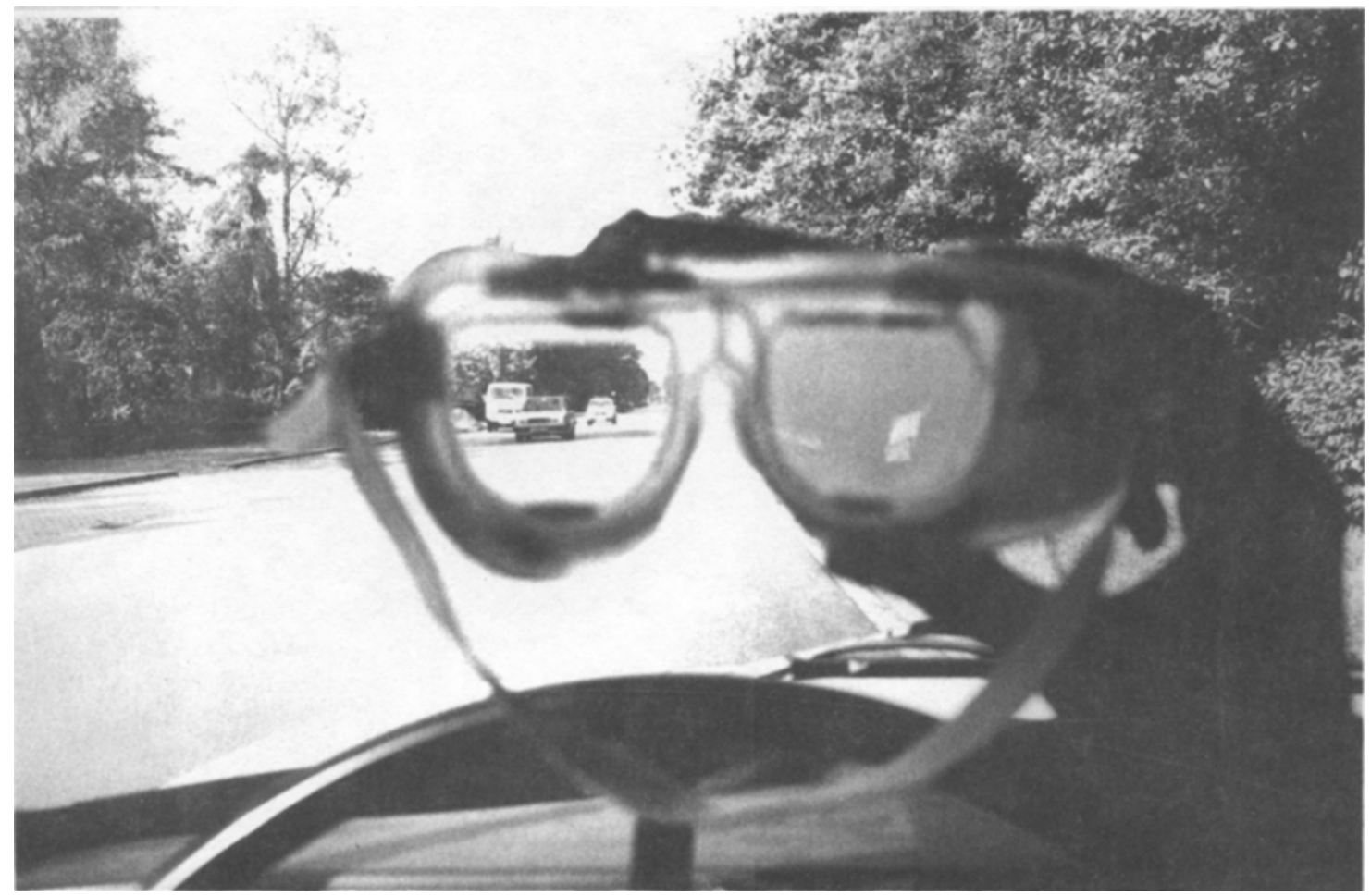

Figure 3. View through PLATO spectacles, in both open and closed states, approximately as seen by automobile driver.

extent to which a particular object being viewed becomes imperceptible due to the scattering of light by the liquid crystals depends on the level of ambient illumination and on the contrast ratio between the luminance of the target object and that of its visual surround: the lower the ambient illumination and the higher the contrast ratio, the more likely the object will be discernible through the glasses in their closed state. Complicating the issue further is the fact that the scattering characteristics of the present liquid crystals vary with time. That is, maximum extinction is reached just after the scattering state initially evolves, but if no further electrical switching is performed within a few seconds the scattering texture gradually disappears, until the cells eventually become almost transparent again. For applications requiring very brief occlusion intervals this is not a major problem, but for applications requiring longer occlusion intervals this may be unacceptable, depending on ambient illumination and target luminance.

It has been found that in general most problems involving insufficient extinction can be alleviated by constructing composite liquid-crystal lenses. These may comprise either separate cells that are stacked in parallel or single cells that share common internal (double-sided) electrode plates. In either case the cumulative light scattering of all of the individual cells during their common closed state acts to increase the effectiveness of the composite device. (The price to be paid for such composite scattering devices clearly is a proportionate increase in total energy con- sumed when switching the cells on.) In Figure 1, for example, the goggle-mounted model shown has been constructed using a triple layer of liquid-crystal cells.

For most practical applications, it should be sufficient to rate the PLATO spectacies' occlusion capabilities as either satisfactory or unsatisfactory, depending on the particular conditions, such as ambient light level, luminance of objects being viewed, duration of occlusion, and so forth. For automobile driving experiments, for example, extinction of relevant visual information has been found to be effectively total for daytime driving. This is illustrated in Figure 3, for double-layered lenses. During nighttime driving, on the other hand, subjects are able to perceive discrete light sources and highly illuminated objects within a few seconds of onset of the closed state; however, most of the essential perceptual cues about the roadway that are necessary for stable automobile control are still effectively occluded.

\section{Electrical and Other Characteristics}

As explained above, application of an (alternating) electric field is necessary to switch the liquid-crystal cells from scattering to transparent. Although all that is needed for any switching is a suprathreshold field, for a fixed cell thickness the time constant for this transition is inversely proportional to the square of the voltage applied (Mollon et al., 1977). (If too large a voltage is applied, however, local sparking across the cell will occur, causing permanent imperfections.) Carrier frequencies of between $1 \mathrm{kHz}$ 
and $100 \mathrm{kHz}$ are usually suitable for the driving voltage. For the 12- $\mu$ cells whose response is shown in Figure 2, for example, a $140-\mathrm{V}-\mathrm{rms}, 2-\mathrm{kHz}$ voltage has been used for switching.

Although relatively high voltage levels may be required to turn a cell on rapidly, it is not necessary to maintain such levels to keep the cell in its transparent state. It is instead possible a short time after switching a cell on, say $4 \mathrm{msec}$, to allow the driving voltage to drop to a lower (but still suprathreshold) level, in the present case about $50-60 \mathrm{~V}$, for the remainder of the duration of the open period. Depending on the duty cycle for the particular application, the total power consumption of the PLATO switching circuitry using this method is along the order of $10 \mathrm{~mW} / \mathrm{cm}^{2}$. With further development efforts, this power consumption figure is expected to decrease (and with it the cost of the switching circuitry), thereby making extended battery operation feasible.

With respect to safety, power consumption with the present device is such that the current to the cells can easily be limited to within acceptable levels. One safety specification that cannot be met with the present device, however, is that it is not strictly fail-safe; that is, if the power supply should fail, the spectacles will remain in their closed rather than open state. For safety-critical applications, such as automobile driving experiments, the best solution to this problem is a judicious combination of (1) ensuring that all conductive elements have been well encased and that all electrical connections are reliable; (2) using pullup amplifiers to interface the switching circuitry to the cell-driving circuitry, so that if the former should fail the cells will remain open; (3) using completely independent power supplies for each eye, since it is much less likely that both sides will fail simultaneously; (4) driving all circuits with batteries, to guard against mains failures; and (5) ensuring that, if necessary, the spectacles can easily and quickly be pulled off by the subject.

With respect to reliability and ruggedness, it has been reported (Clark, 1979) that 90\%-95\% of such liquidcrystal devices may be expected to operate for 10 years or more. It also has been found (through inadvertent experimentation) that the prototype spectacles are quite rugged, having been able to survive falls from about $2 \mathrm{~m}$ onto hard surfaces. It is also quite feasible to encase each of the cells, or combinations of cells, within a protective layer of glass or clear plastic. In such instances, it is important to ensure that no undesirable effects arise due to impaired heat conduction.

Temperature of operation is an important parameter to consider when deciding upon the suitability of the PLATO device. Within the range of operating temperatures that one might expect to encounter during normal experimentation, say $15^{\circ}-30^{\circ} \mathrm{C}$, no problems have been encountered thus far. Since the clearing point of cholesteric mixtures is approximately $60^{\circ} \mathrm{C}$, care should be taken not to let the cells approach this temperature. (No damage to the cells occurs at such temperatures; the result is merely constant transparency.) Such an overheating ef- fect may quite possibly occur if the cells are left on with a high driving voltage for long periods of time, especially with composite cells, which are less able to rid themselves of excess heat. However, with the circuitry described above, whereby during each switching cycle the voltage is reduced after a few milliseconds, the likelihood of overheating is greatly diminished. At the opposite extreme, the melting point of the host eutectic is about $-10^{\circ} \mathrm{C}$, so very low temperatures also should be avoided. Other thermal effects may include variation of the magnitude of the threshold switching field and changes in the viscosity of the fluid mixtures, where decreasing temperatures lead to higher viscosities and slower responses (Clark, 1979).

\section{APPLICATIONS}

As mentioned above, the PLATO spectacles were developed originally for the purpose of investigating certain aspects of visual information processing performance during automobile driving, by totally occluding a driver's visual field and allowing him/her only selected, controlled glimpses of the roadway. The driver is thereby constrained to using the minimal amount of (otherwise highly redundant) visual information necessary for safely controlling the vehicle. This use of visual occlusion as a human performance research tool was apparently first applied by Senders et al. (1967), whose experiments were based on earlier theories of man-machine systems modeling.

Senders et al.'s (1967) approach utilizes the notion that it is possible to estimate the attentional demand, or information processing workload, imposed on a human monitor/controller of a (complex) system by recording the circumstances and rate at which he/she samples information from the system. A more conventional, related technique for assessing an operator's interaction with the visual environment is to measure the subject's eye point-of-regard. The visual-occlusion approach assumes, however, that, rather than attempting to record in detail when and where an operator has directed his/her gaze, it is often useful to know simply how frequently and/or how long he/she has elected to observe a particular display or visual environment to acquire the information that is necessary for monitoring or controlling the associated system satisfactorily. Given the well-known difficulties associated with recording and interpreting eye point-of-regard data, the visual-occlusion method can in many cases be a straightforward and economical alternative means of acquiring insight into information processing demands and operator performance.

Since the original experiments of Senders et al. (1967), a number of other automobile driving experiments using visual occlusion have been reported, including those of Zwahlen and Balasubramanian (1974), a theoretical and experimental investigation of drivers' uncertainty while steering with no visual input; Triggs and Caple (1978), an investigation of driver information processing, using visual occlusion to discriminate driving performance in 
night versus daytime conditions; Fraser and Perry (1980), an investigation of the feasibility of using visual occlusion for studying visual sampling behavior in drivers; Triggs and Berenyi (1982), estimation of vehicle speed using tachistoscopic observations; Milgram, Godthelp, and Blaauw (1982), an investigation of drivers' decisionmaking criteria with respect to the sampling of visual information; Blaauw, Godthelp, and Milgram (1984), mathematical modeling of observation and control in automobile driving as a supervisory control task; Godthelp (1986) and Godthelp, Milgram, and Blaauw (1984), verification of a preview prediction model of control strategy in automobile driving.

The range of human performance research areas for which the use of interrupted vision is a useful experimental tool can clearly be extended, within the broader context of visual-motor coordination in general, to include topics ranging from highly skilled performance, such as that of aircraft pilots or professional athletes, to such common daily activities as "simple" locomotion and obstacle avoidance (e.g., Thomson, 1980). For example, in a number of earlier investigations into the relationship between skill level, vision and proprioception during ball catching, experimenters have employed a variation of the visual-occlusion method by controlling room lighting during the interval in which balls were projected at experimental subjects (e.g., Sharp \& Whiting, 1974). The advantages of using PLATO spectacles in such experiments, instead of flashing room lights on and off, especially with respect to potential problems of the eye's having to adapt to darkness and light, are obvious. Furthermore, the possibility of selectively occluding a subject's peripheral field (e.g., Fischman \& Schneider, 1985) should be made possible, as mentioned earlier, by the development of goggles with switchable liquid-crystal lenses mounted within the goggle walls.

Applications are also foreseen in certain areas of basic perceptual research, such as movement perception. For example, using the device as a stroboscope, by varying the frequency and duration of repetitive visual field exposures, it should be possible to influence one's sensation of apparent (as opposed to real) movement (Anstis, 1978) and thus investigate a wide range of psychophysical phenomena with ambulatory subjects, both in the laboratory and, as indicated above, during the performance of (complex) psychomotor tasks in the field (e.g., Riemersma, 1982).

In none of the above application areas was mention made of any differences between the stimuli presented to the two eyes of the observer. In research on binocular vision, however, it is usually necessary to dissociate the visual inputs to the eyes. This can of course be accomplished with conventional tachistoscopes, or with devices such as mechanical phase difference haploscopes, halfsilvered mirrors, polarizing spectacles, anaglyph glasses, and so forth, each of which has its own characteristic advantages and shortcomings. Such applications often re- quire only that each eye be exposed to the same source of stimulation, such as light flashes or a checkerboard pattern, at different times. Dissociation can also be achieved with the PLATO device, however, by alternately switching each side of the spectacles in synchrony with a compatible pair of (rapidly) alternating display sources, thereby providing each eye essentially simultaneously with different, independently controllable display information. (See Stereoscopic Displays below.)

Finally, the capability of dissociating binocular inputs by means of visual occlusion is of potential significance also for a number of clinical ophthalmological applications. In recent years, visual evoked potentials (VEPs) to pattern stimuli have emerged as an effective clinical probe, as an alternative to earlier, more tedious preferential looking techniques, for purposes such as the testing of visual acuity in infants (Spekreijse, 1983) and the early identification of such anomalies as amblyopia and albinism (Apkarian, Reits, \& Spekreijse, 1984). During VEP testing the examination of each eye individually conventionally involves placing a patch or bandage over the nontested eye, a procedure that can be disturbing for very young children. The use of (specially adapted) electrooptic shuttering spectacles shows some promise for facilitating this procedure, however, since not only would eye patches no longer be necessary, but also new methods of displaying and temporally ordering the stimuli presented to the two eyes would be realizable. For example, instead of testing each of a patient's eyes sequentially, by physically moving a patch after each test phase, it should be possible to shorten the entire examination procedure by testing both eyes essentially at the same time, by means of alternately or randomly varying stimulus presentation from one eye to the other.

\section{STEREOSCOPIC DISPLAYS}

An additional important application of the PLATO spectacles, also involving the dissociation of visual inputs to the observer's eyes, is as a viewing apparatus for fullcolor field-sequential stereoscopic displays. The fieldsequential method of stereoscopy is currently one of the most economical and easily implementable means of presenting three-dimensional (3D) visual images stereoscopically with standard display equipment. These may be either direct or recorded video images ("3D TV") or computer-generated graphic images.

The principle behind field-sequential, or timemultiplexed, stereoscopic displays involves rapidly and alternately presenting left- and right-eye perspective images on a display surface, such as a CRT or projection screen. The observer views these images through an electronically driven shutter device, such as PLATO, which separately and alternately blocks and unblocks the view of the display from each eye, in synchrony with the alternating display images. The result is that the left eye is blocked whenever the right eye (right camera) image 
is presented and unblocked whenever the left eye (left camera) image is presented. The converse pertains for the right eye.

A more detailed description of the use of PLATO spectacles as a stereoscopic viewing device, in particular in comparison with other electrooptic shuttering devices that have been used for similar purposes, is presented elsewhere (Milgram \& van der Horst, 1986). The principal problem associated with other viewing devices, most of which use polarizers to selectively transmit or extinguish incident light, is a significant reduction in display image brightness, which can only partly be compensated for by increasing the display gain. As explained above, PLATO's use of light-scattering cholesteric liquid crystals to occlude the observer's visual field obviates the need for polarizers, thereby resulting in a much higher level of light transmission when the spectacles are in the open state.

The applications of stereoscopic displays are many and well known. Some of the more prominent of these include: teleoperation of remote vehicles/manipulators (e.g., for space, airborne, underwater, land, nuclear applications), flight simulation (e.g., nap-of-the-earth flight, formation flying, mid-air refueling), automobile driving simulation, air traffic control displays, computer graphics and computer-aided design, molecular modeling, and medical imaging.

\section{CONCLUSION}

The liquid-crystal device described in this paper is not the only spectacle-mounted electrooptic shuttering apparatus to appear in the literature. Most devices, which can be categorized as either light-scattering or lightextinguishing, differ from the PLATO spectacles in a number of important respects, however.

Most of the light-scattering devices (e.g., the liquidcrystal tachistoscope of Moukhwas \& Perrot, 1978) operate on the basis of dynamic scattering, rather than the cholesteric-nematic phase change effect described here. One potential advantage of dynamic-scattering devices is the ability to vary the degree of fogginess of the lenses continuously between a minimum (transparent) and a maximum by raising or lowering the driving voltage. Some practical applications of that property, for example, include the development of a "cloud simulator" for pilot flight training applications (Witt, 1978) and an experimental investigation of visual cues used by pilots during helicopter hovering tasks (Hoh, 1985). The principal shortcoming of those liquid-crystal shutters, however, is their relatively long switching times, along the order of tens of milliseconds, which obviously preclude their use for most tachistoscopic experiments in visual perception.

Many of the shuttering devices in the light-extinguishing category have been developed primarily for fieldsequential stereoscopic display applications, where, in order to function satisfactorily, they must be able to switch very rapidly, at least 25-30 complete open-close cycles per second (a requirement that cannot be met by the dynamic-scattering devices cited above). Among the earliest light-extinguishing electrooptic shutters applied to field-sequential stereoscopy were conventional twistednematic liquid crystals (Roese, 1977). These devices were too slow for the intended application, however, with relaxation times greater than $10 \mathrm{msec}$ (which necessitated the blanking of large portions of each display field to prevent cross talk between sequential left and right images). The original twisted-nematic devices were thus eventually supplanted by much faster PLZT (polycrystalline lanthanum-doped lead-zirconate-titanate) ceramics (Roese \& Khalafalla, 1976). PLZT devices also possess some serious drawbacks, however, one of which is the rather high voltage required to operate them $(500-\mathrm{V} \mathrm{dc}, 15-\mathrm{W}$ peak transient power). The recent development of newer, low-power devices, such as pi-cells (Bos, Johnson, \& Koehler/Beran, 1983) and "two-frequency" switched nematic crystals (Shanks, 1975), on the other hand, have once again made the use of liquid crystals attractive for stereoscopic applications.

All of the light-extinguishing shuttering devices mentioned thus far share the common property of employing front and rear (crossed) polarizers to extinguish incident light when the shutters are in their closed state. The consequence of this, however, is a severe reduction in light transmission when the electrooptic shutters are in their open state. For PLZT devices, for example, open-state light-attenuation factors of $83 \%-85 \%$ have been cited (Roese \& Khalafalla, 1976). For many stereoscopic displays and ophthalmological applications, the severe reduction in display image brightness that results when lightextinguishing spectacles are used is usually a significant disadvantage (Milgram \& van der Horst, 1986), which can have consequences also for most of the tachistoscopic presentation applications outlined above.

In conclusion, therefore, although possibly not as rapid as the PLZT or the newer liquid-crystal devices, the lightscattering PLATO spectacles nevertheless offer a number of significant attractive and demonstrable features, including low cost, reliability, safety, minimal reduction in the brightness of the viewer's visual input, and, especially, a wide diversity of applications both within and beyond the laboratory.

\section{REFERENCES}

ANsTIS, S. M. (1978). Apparent movement. In R. H. Held \& H. W. Leibowitz (Eds.), Handbook of sensory physiology: Vol. 8. Perception (pp. 655-673). NY: Springer-Verlag.

ApKarian, P., ReITs, D. \& SPekreuse, H. (1984). Component specificity in albino VEP asymmetry: Maturation of the visual pathway anomaly. Experimental Brain Research, 53, 285-294.

Blaauw, G. J., Godthelp, H., \& Milgram, P. (1984). Optimal control model applications and field measurements with respect to car driving. Vehicle System Dynamics, 13(2), 99-111.

Bos, P. J., Johnson, P. A., \& Koehler/Beran, K. R. (1983). A liquid crystal optical switching device (pi-cell). SID Digest, 30-31.

ClarK, M. G. (1979). Liquid crystal displays and colour switches. Displays: Technology \& Applications, 1(1), 17-24.

Fischman, M. G., \& SCHNEIDER, T. (1985). Skill level, vision, and 
proprioception in simple one-hand catching. Joumal of Motor Behavior, 17, 219-229.

Fraser, P. J., \& PERry, D. R. (1980). The ARRB visual interruption apparatus. (Technical Manual ATM, No. 11). Victoria, Australia: Australian Road Research Board.

GoDTHELP, H. (1986). Vehicle control during curve driving. Human Factors, 28, 211-221.

Godthelp, H., Milgram, P., \& Blaauw, G. J. (1984). The development of a time-related measure to describe driving strategy. Human Factors, 26, 257-268.

GreEN, B. F. (Ed.). (1978). Computer-based tachistoscopes (Special section). Behavior Research Methods \& Instrumentation, 10, 754-795.

HoH, R. H. (1985). Investigation of outside visual cues required for low speed and hover. Proceedings of 12th ALAA Atmospheric Flight Mechanics Conference. New York: American Institute of Aeronautics and Astronautics (Rep. No. ALAA-85-1808).

Milgram, P., Godthelp, H., \& BlaAuw, G. J. (1982). An investigation of decision-making criteria adopted by drivers while monitoring vehicle state in the temporary absence of visual input. Second European Annual Conference on Human Decision Making and Manual Control (pp. 104-116). Bonn, West Germany: University of Bonn and Forschungsinstitut für Anthropotechniek.

Milgram, P., \& VAN DER Horst, R. (1986). Alternating-field stereoscopic displays using light-scattering liquid crystal spectacles. Displays: Technology \& Applications, 7(2), 67-72.

Mollon, J. D., Polden, P. G., \& Morgan, M. J. (1977). Electrooptic shutters and filters. Quarterly Journal of Experimental Psychology, 29, 147-156.

Moukhwas, D., \& Perrot, C. (1978). A new device for research on visual perception: The liquid crystals tachistoscope. Ergonomics, 21, 309-312.

RaYNes, E. P. (1976). Cholesteric texture and phase change effects. In A. R. Kmetz \& F. K. von Willisen (Eds.), Nonemissive electrooptic displays (pp. 25-43). New York: Plenum Press.

Riemersma, J. B. J. (1982). Perceptual cues in vehicle guidance on a straight road. Second European Annual Conference on Human Decision Making and Manual Control (pp. 127-136). Bonn, West Germany: University of Bonn and Forschungsinstitut für Anthropotechniek..

RoEse, J. A. (1977). Liquid crystal stereoscopic viewer. U.S. patent $4,021,846$.

Roese, J. A., \& Khalafalla, A. S. (1976). Stereoscopic viewing with PLZT ceramics. Ferroelectrics, 10, 47-51.
Senders, J. W., Kristofferson, A. B., Levison, W. H., Dietrich, C. W., \& WARD, J. L. (1967). The attentional demand of automobile driving. Highway Research Record, 195, 15-33.

SHaNKs, I. A. (1975). Multicolour displays using a liquid crystal colour switch. AGARDograph, 167, 18.1-11.

Sharp, R. H., \& WhrtiNG, H. T. A. (1974). Exposure and occluded duration effects in a ball-catching skill. Journal of Motor Behavior, 6, 139-147.

SPEKREIJSE, H. (1983). Comparison of acuity tests and pattern evoked potential criteria: Two mechanisms underly acuity maturation in man. Behavioural Brain Research, 10, 107-117.

Thомson, J. A. (1980). How do we use visual information to control locomotion? Trends in Neurosciences (TINS), 3, 247-250.

TrigGS, T. J., \& BERENYI, J, S. (1982). Estimation of automobile speed under day and night conditions. Human Factors, 24, 111-114.

Triggs, T. J., \& CAPLE, D. C. (1978). Visual sampling by drivers in night versus day conditions. Proceedings of 15th Annual Human Factors Conference of Australia \& New Zealand. Melbourne, Australia: University of Melbourne.

WITT, F. A. (1978). Head mounted flight training apparatus. U.S. patent $4,106,217$.

ZWAHLEN, H. T., \& Balasubramanian, K. N. (1974). A theoretical and experimental investigation of automobile path deviations when driver steers with no visual input. Transportation Research Record, 520, 25-37.

\section{NOTE}

1. Because it was impractical to order the construction of unit quantities of customized liquid-crystal cells of the required size, shape, and composition for purposes of experimentation, the cells were fabricated in the author's laboratory using the cholesteric biphenyl CB15 in admixture with nematic E18, both purchased from BDH Ltd. of Poole, England. Since, at the time of this publication, production of the spectacles has not been undertaken on a commercial basis, this do-it-yourself option is, in the author's opinion, still the most economical means at the present time of obtaining such spectacles for research purposes.

(Manuscript received December 1, 1986; revision accepted for publication June 2, 1987.) 